\title{
PREVALENCIA DE MEMBRANAS EPIRRETINIANAS MEDIANTE TOMOGRAFÍA DE COHERENCIA ÓPTICA EN PACIENTES REMITIDOS PARA CIRUGÍA DE CATARATAS
}

\section{USE OF OPTICAL COHERENCE TOMOGRAPHY TO MEASURE PREVALENCE OF EPIRETINAL MEMBRANES IN PATIENTS REFERRED FOR CATARACT SURGERY}

\author{
CONTRERAS $\mathrm{I}^{1}$, NOVAL $\mathrm{S}^{1}$, TEJEDOR $\mathrm{J}^{2}$
}

\section{RESUMEN}

Objetivo: Evaluar la prevalencia de membranas epirretinianas (MER) en pacientes remitidos para cirugía de cataratas y la frecuencia de aparición de nuevas MER en los primeros 6 meses tras la facoemulsificación mediante tomografía de coherencia óptica (OCT).

Métodos: La prevalencia de MER en pacientes referidos para cirugía de catarata se determinó a partir de una evaluación oftalmológica inicial completa que incluía una exploración con OCT. Todos los pacientes fueron operados mediante facoemulsificación sin complicaciones. Si existía una MER en un ojo, el contralateral era programado para cirugía de catarata. Si no se detectaba MER en ninguno, se operaba el ojo con peor agudeza visual. Los pacientes fueron seguidos durante 6 meses para estudiar la influencia de la facoemulsificación en el desarrollo de MER.

Resultados: Cuarenta y cinco pacientes fueron incluidos en el estudio. En la evaluación inicial, se detectó la presencia de MER mediante OCT en siete pacientes $(15,6 \%)$. Una paciente presentaba una MER en ambos ojos, siendo visible mediante funduscopia sólo en el ojo izquierdo. Las MER no eran

\section{ABSTRACT}

Purpose: To evaluate, using optical coherence tomography (OCT), the prevalence of epiretinal membranes (ERM) in patients referred for phacoemulsification, and the frequency of new ERM development in the first six months after surgery.

Methods: Patients referred by general ophthalmologists for cataract surgery underwent a new, complete ophthalmological evaluation. OCT scanning was performed using the Macular Thickness Map acquisition protocol (StratusOCT, Carl Zeiss Meditec). This baseline examination was used to determine the prevalence of ERM in patients referred for cataract surgery.

All patients underwent uneventful phacoemulsification. If an ERM was already present in one eye, the other eye was chosen for cataract surgery. If no ERM was present, the eye with the poorest visual acuity was operated. Operated eyes were followedup for six months in order to study the influence of phacoemulsification on the development of ERM.

Results: Forty-five patients were prospectively evaluated. In the baseline visit, ERM were detected by OCT in 7 patients (15.6\%). Both eyes were affected in one patient, with the ERM being apparent on fun-

\footnotetext{
Recibido: 29/1/07. Aceptado: 17/1/08.

Hospital Universitario Ramón y Cajal. Madrid. España.

1 Licenciado en Medicina.

2 Doctor en Medicina.

Correspondencia:

Inés Contreras

C/. Martín de los Heros, $80,5^{\circ} \mathrm{D}$

28008 Madrid

España

E-mail: inescon3@yahoo.com
} 
evidentes en la exploración del fondo de ojo en los demás casos.

Tras 6 meses de seguimiento, no se detectaron nuevos casos de MER en los ojos operados, ni mediante evaluación del fondo de ojo ni mediante OCT.

Conclusión: Existe la posibilidad de que un elevado porcentaje de las MER que se consideran secundarias a la cirugía de cataratas estén presentes antes de la misma, pudiendo ser diagnosticadas mediante OCT.

Palabras clave: Cirugía de catarata, facoemulsificación, membranas epirretinianas, efectos secundarios. duscopy only in her left eye. Fundus examination was unremarkable in all other cases.

After six months follow-up, no new cases of epiretinal membranes were detected in the eyes that had undergone phacoemulsification, neither by fundus evaluation nor by OCT.

Conclusions: Epiretinal membranes, previously thought to be secondary to cataract surgery, may be present before phacoemulsification and can be diagnosed by OCT (Arch Soc Esp Oftalmol 2008; 83: 89-94).

Key words: Optical coherence tomography, epiretinal membrane, phacoemulsification, cataract surgery, side-effects.

\section{INTRODUCCIÓN}

Las membranas epirretinianas (MER) se caracterizan por la proliferación de tejido anómalo sobre la superficie de la retina. Pueden aparecer en asociación con diversas patologías oculares (enfermedades vasculares retinianas, tumores intraoculares, enfermedades oculares inflamatorias, traumatismos oculares, desprendimientos de retina...) o tras cirugías oculares (1-3). Las MER que se desarrollan en ojos sanos sin existir ninguna anomalía ocular ni historia previa de enfermedades asociadas se denominan MER idiopáticas.

Se cree que las MER se desarrollan a partir de células gliales retinianas que migran a la superficie de la retina a través de roturas en la membrana limitante interna (MLI) (4). El desprendimiento de vítreo posterior (DVP) podría estar implicado en la patogénesis de las MER idiopáticas, al producir desgarros en la MLI; esta teoría se ve apoyada por numerosos estudios que han encontrado una prevalencia de DVP mucho mayor en pacientes con MER frente a poblaciones del mismo sexo y edad (1,4-8).

Clínicamente, las MER comienzan como un reflejo luminoso cambiante en oftalmoscopia (9), produciendo escasos o ningún cambio en la superficie retiniana. En esta etapa, denominada «reflejo celofánico macular» (RCM), el paciente habitualmente no presenta síntomas (10). A medida que la enfermedad avanza, la capa prerretiniana de células se engruesa, contrayéndose posteriormente, lo que conduce al desarrollo de pliegues retinianos. Este tipo de MER, en la cual el reflejo luminoso se con- vierte en opaco y gris, se denomina «fibrosis premacular» (FPM). Los pacientes pueden presentar pérdida de agudeza visual (AV) o metamorfopsias.

Recientemente se han publicado diversos estudios poblacionales que investigaban la prevalencia de las MER. En estos estudios, las MER se diagnosticaban principalmente a través de la evaluación de retinografías; en algunos, el fondo de ojo también era examinado mediante biomicroscopía. Las tasas de prevalencia oscilan entre un $2,8 \%$ en poblaciones japonesas y un $19,0 \%$ en latinos de los EEUU (2,3,11-14).

La cirugía de cataratas se considera una de las causas de MER secundarias, debido a que estudios transversales y retrospectivos han encontrado una mayor prevalencia de MER en pacientes afáquicos y pseudofáquicos. Sin embargo, publicaciones recientes han cuestionado que la facoemulsificación no complicada pueda inducir la formación de MER (15).

El objetivo de este estudio es evaluar la prevalencia de MER en pacientes referidos para cirugía de cataratas y la frecuencia de aparición de nuevas MER durante los primeros 6 meses tras la cirugía mediante la realización de exploraciones periódicas con tomografía de coherencia óptica (OCT).

\section{SUJETOS, MATERIAL Y MÉTODOS}

Todos los pacientes referidos para cirugía de cataratas a la consulta de uno de los autores (JT) entre abril y junio de 2005 fueron evaluados. Los pacientes habían sido estudiados previamente por un oftal- 
mólogo general que había determinado que la presencia de cataratas era la causa de la pérdida visual. Todos los pacientes fueron sometidos a una nueva exploración oftalmológica completa, incluyendo AV, biomicroscopía con valoración de la catarata mediante la clasificación LOCS III [Lens Opacities Classification System III (16)], tonometría de aplanación, oftalmoscopía indirecta y evaluación biomicroscópica del polo posterior con lente de no contacto. La exploración con OCT se realizó utilizando el protocolo de adquisición Macular Thickness Map (StratusOCT, Carl Zeiss Meditec, Dublín, CA, EEUU). Los pacientes fueron excluidos del estudio si presentaban cualquier patología ocular salvo escasas drusas maculares, DVP o cambios inespecíficos leves del epitelio pigmentario de la retina. También se excluyó a aquellos pacientes que tenían cataratas tan densas que no permitían obtener una señal clara en la OCT y a aquellos con una longitud axial más allá de la capacidad de enfoque de la OCT. Todos los pacientes firmaron un consentimiento informado tras conocer las características del estudio.

La prevalencia de MER en pacientes derivados para cirugía de cataratas se obtuvo a partir de esta exploración inicial. Si ya existía una MER en un ojo, el otro ojo era el programado para cirugía de catarata. Si no existían MER, se programaba para cirugía el ojo con peor AV. Se siguió a los pacientes durante 6 meses después de la cirugía para estudiar la influencia de la facoemulsificación en el desarrollo de MER.

La cirugía de catarata se realizó a través de una incisión temporal en córnea clara. Tras la facoemulsificación, se introdujo una lente de silicona plegable (Cee-on Edge ${ }^{\circledR}$, AMO) en el saco capsular y se inyectó $1 \mathrm{mg}$ de cefuroxima en cámara anterior (Curoxima ${ }^{\circledR}$, Roche). Los pacientes fueron evaluados el día después de la cirugía, así como 2, 6, 12 y 24 semanas después de la misma. En todas las visitas se realizó una valoración oftalmológica completa, que incluía la exploración con OCT en las semanas 6,12 y 24 .

El protocolo de adquisición Macular Thickness Map realiza seis barridos radiales centrados en la fóvea, localizada mediante fijación interna. Todas las exploraciones fueron realizadas por dos de los autores (IC, SN) tras midriasis farmacológica, grabándose tan sólo las imágenes que cumplían criterios subjetivos de calidad. Además, antes de aceptar una exploración como válida para el análisis, se procedía a efectuar una evaluación mediante el protocolo Retinal Thickness Analysis para comprobar que el software del OCT había identificado correctamente las distintas capas retinianas en al menos cinco de los seis barridos. Si los bordes retinianos estaban delineados de forma incorrecta en un único barrido, éste era eliminado manualmente del análisis; en caso contrario, se eliminaba todo el protocolo de adquisición y se repetía la exploración. Cada imagen era estudiada por los autores para determinar si existía una MER. El volumen macular y el espesor retiniano medio de un área de $1 \mathrm{~mm}$ de diámetro centrada en la fóvea (espesor macular central) fueron obtenidos mediante el protocolo de análisis Retinal Thickness/Volume.

El estudio estadístico se realizó con el programa SPSS 12.0 (SPSS for Windows, SPSS Inc, Chicago, Illinois). Se emplearon pruebas no paramétricas con todas las variables. La AV fue convertida a la escala $\log$ MAR para el análisis estadístico. La AV, el espesor macular central y el volumen macular de los distintos grupos fueron comparados mediante la prueba de Mann-Whitney. Las correlaciones entre AV y las medidas tomográficas se realizaron con la prueba de correlación de Spearman.

\section{RESULTADOS}

Cuarenta y cinco pacientes fueron incluidos en el estudio; 17 varones y 28 mujeres, con una edad mediana de 73 años (rango de 29 a 88 años). En la visita inicial, se detectaron MER mediante la OCT en siete pacientes $(15,6 \%)$. En una paciente existía una afectación bilateral, siendo tan sólo la MER evidente en la exploración de fondo de ojo en el ojo izquierdo (fig. 1). La exploración del fondo de ojo no mostraba cambios destacables en el resto de los casos. Las MER aparecían en la OCT como una fina banda hiperreflectiva en la superficie de la retina, que producía cambios en el contorno foveal (fig. 2).

La AV basal de todos los ojos que fueron posteriormente operados, así como de los ojos adelfos con y sin MER se muestran en la tabla I. Entre los ojos no operados, los ojos con MER tenían una peor AV que los ojos sin MER ( $p=0,027$, tabla I). La paciente con MER bilateral no está incluida en la tabla I. En esta paciente, la AV mejoró de 0,7 a 0,4 unidades $\log$ MAR en el ojo operado. La AV mejoró en el resto de los ojos operados de 0,80 a 0,42 unidades $\log$ MAR $(\mathrm{p}<0,001)$.

El espesor macular central fue significativamente mayor en ojos con MER: 334,71 $\mu \mathrm{m}$, desviación 


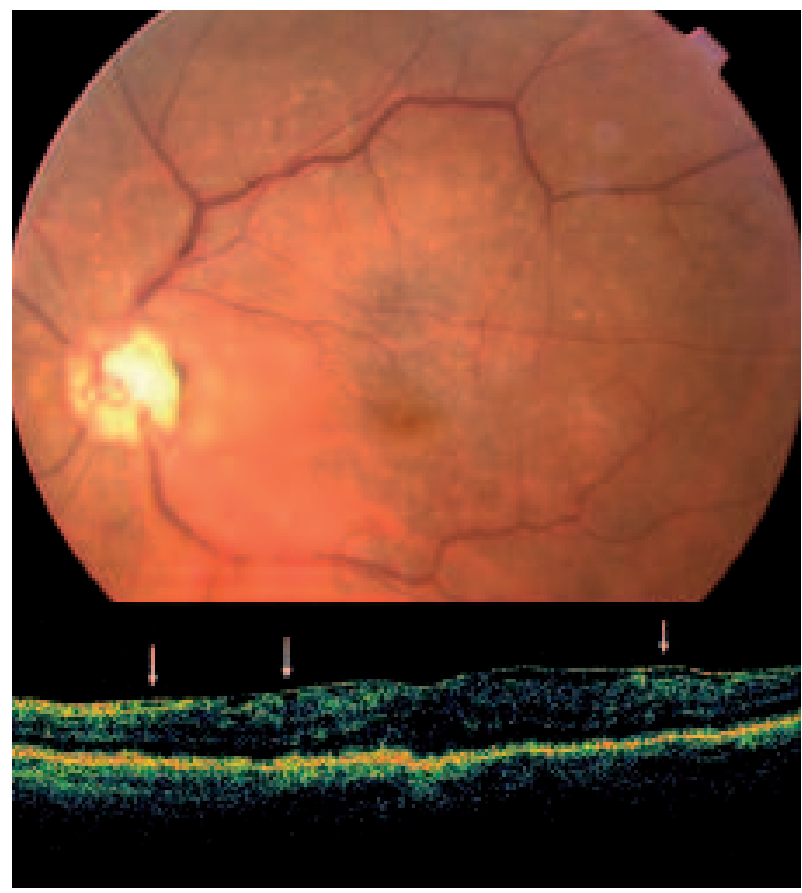

Fig. 1: Retinografía y tomografía de coherencia óptica del único paciente en el que la membrana epirretiniana era evidente en el examen del fondo de ojo. La membrana epirretiniana aparece como una fina banda hiperreflectiva (flechas).

estándar (DE) 92 versus 211,16 um DE 27,12; $\mathrm{p}<0,001$. Seis pacientes con MER presentaban espesores maculares centrales mayores de $250 \mu \mathrm{m}$. El volumen macular también era significativamente mayor en ojos con MER: 8,26 $\mathrm{mm}^{3}$, DE 1,08 versus $6,89 \mathrm{~mm}^{3}$, DE $0,5(\mathrm{p}<0,01)$. No se encontraron correlaciones estadísticamente significativas entre la AV y el espesor macular central ni entre la AV y el volumen macular.

Tras 6 meses de seguimiento, no se detectaron nuevos casos de MER en los ojos que habían sido

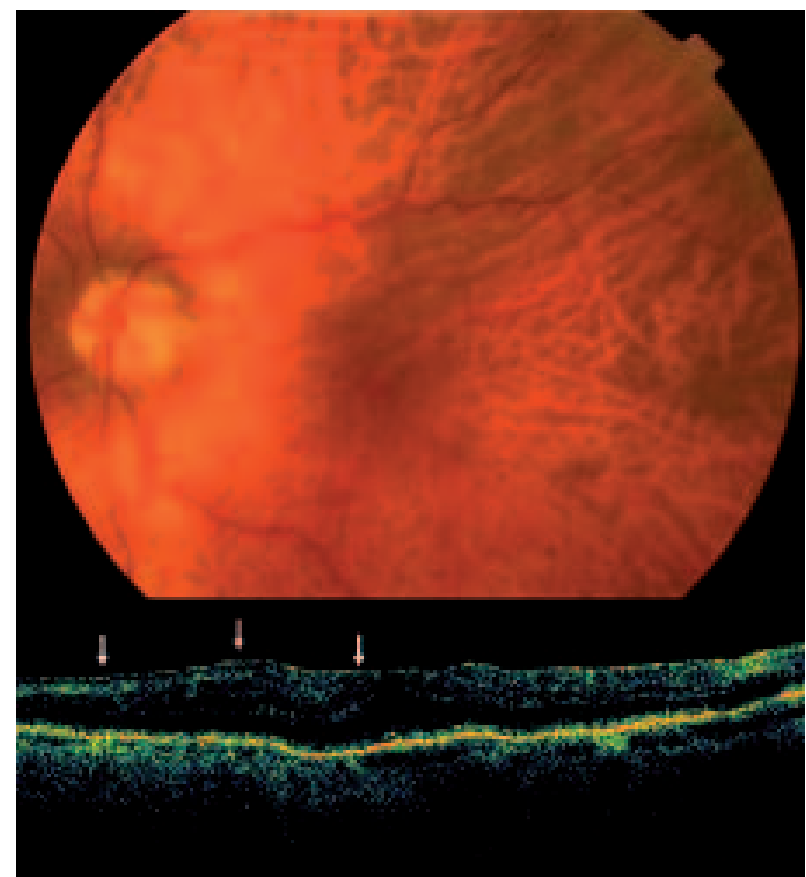

Fig. 2: Retinografía y tomografía de coherencia óptica de uno de los pacientes con una membrana epirretiniana diagnosticada por tomografía de coherencia óptica, no evidente en la exploración funduscópica. La membrana epirretiniana aparece como una fina banda hiperreflectiva (flechas).

operados de catarata, ni mediante exploración del fondo de ojos ni mediante OCT. Tampoco se diagnosticaron nuevas membranas en este periodo en el ojo adelfo no operado.

\section{DISCUSIÓN}

Recientemente diversos estudios poblacionales han intentado determinar la prevalencia de MER. La mayoría de estos estudios consideran que las

Tabla I. Agudeza visual en los ojos operados y adelfos a lo largo del seguimiento

\begin{tabular}{lcccc}
\hline & $\begin{array}{c}\text { AV ojo operado } \\
\text { Snellen } \\
\text { Media (DE) }\end{array}$ & Todos & $\begin{array}{c}\text { AV ojo adelfo } \\
\text { Snellen } \\
\text { Media (DS) } \\
\text { Sin MER }\end{array}$ & Con MER \\
\hline Visita & $0,41(0,20)$ & $0,65(0,28)$ & $0,69(0,28)$ & $0,43(0,19)$ \\
Basal & $0,74(0,18)$ & $0,67(0,27)$ & $0,71(0,25)$ & $0,46(0,26)$ \\
6. ${ }^{\text {s semana }}$ & $0,78(0,18)$ & $0,60(0,29)$ & $0,66(0,28)$ & $0,31(0,12)$ \\
24 semana & $0,79(0,16)$ & $0,68(0,27)$ & $0,73(0,26)$ & $0,42(0,25)$ \\
\hline \hline
\end{tabular}

AV agudeza visual; DE desviación estándar. 
MER diagnosticadas en pacientes pseudofáquicos son membranas secundarias. Las tasas de prevalencia de estos estudios difieren notablemente $(2,3,11$ 14). Las MER se clasifican como reflejo celofánico macular (RCM) o fibrosis premacular (FPM). Un análisis más detallado demuestra que la prevalencia de FPM es similar en todos estos estudios (con un rango de entre 1,1 y 1,9\%), mientras que la tasa de RCM es la principal fuente de variación.

McCarty et al especularon con la posibilidad de que las diferencias en la tasa de RCM se debiesen a diferentes sistemas de evaluación (número de fotografías de fondo de ojo, fotografías estereoscópicas) o a la diferencia en la pigmentación del fondo de ojo, que harían que el RCM fuese más visible en poblaciones hispanas (11). Al analizar las características de las membranas secundarias, se puede ver que en causas distintas de la cirugía de cataratas, como la cirugía del desprendimiento de retina o la oclusión de vena retiniana, la FPM es más frecuente que el RCM. Sin embargo, la proporción de RCM frente a FPM en MER diagnosticadas tras la cirugía de cataratas es muy similar a la presente en membranas idiopáticas $(2,12)$.

La cirugía de cataratas ha sido considerada tradicionalmente una causa de MER debido a que diversos estudios de prevalencia de MER encontraron valores más altos en pacientes pseudofáquicos (13,11-13). El diagnóstico de MER en estos estudios está basado en retinografías y sólo ocasionalmente se confirmó mediante una exploración con lente. De este modo, se puede plantear que el diagnóstico de una MER pre-existente podría infravalorarse en pacientes con cataratas en los que con frecuencia es difícil obtener una imagen clara y bien definida del fondo de ojo (16). Otro factor de confusión podría ser el hecho de que los pacientes con MER pueden tener peores AV que aquellos que no las padecen aumentando la probabilidad de que se les derive para cirugía de catarata.

Hasta el 95\% de los pacientes con MER idiopáticas presentan un DVP, una prevalencia mucho mayor que en otras poblaciones de edad similar $(1,4,6)$. Por ello, se considera que es un mecanismo patogénico de las MER, puesto que al desprenderse el vítreo se pueden producir desgarros en la MLI a través de los cuales las células gliales pueden alcanzar la superficie de la retina. También se cree que la cirugía de catarata puede conducir a la formación MER debido a la inducción quirúrgica de un DVP al traccionar sobre la interfase vítreorretiniana (15).
Sin embargo, no encontramos ningún estudio publicado que analice la incidencia de DVP tras la cirugía de catarata.

Jahn y colaboradores analizaron la incidencia de MER tras la cirugía de cataratas de forma novedosa (15). Los pacientes fueron evaluados antes y menos de 2 semanas después de la cirugía, considerándose esta última la exploración basal. Se encontró que la prevalencia de MER era aproximadamente el doble una vez que se podía obtener una imagen nítida del fondo de ojo. Esto sugiere que las MER pueden pasar desapercibidas con facilidad en presencia de cataratas, ya que 2 semanas es un periodo demasiado corto como para pensar que estas MER no existían previamente a la cirugía. Este estudio también encontró que la prevalencia de MER aumentaba de un $14,8 \%$ (evaluación basal) a un $25,3 \%$ a los 6 meses y a un $27,3 \%$ a los 12 meses de la cirugía. Todos los casos diagnosticados de novo fueron RCM.

El principal problema de los estudios realizados hasta la fecha es que el diagnóstico de MER se basa en una evaluación subjetiva. A menudo, la presencia o ausencia de un reflejo celofánico es difícil de determinar; más aún si la imagen no es nítida debido a la presencia de cataratas. La OCT permite obtener imágenes de la retina con una resolución axial de $10 \mu \mathrm{m}(17,18)$. Aunque la presencia de cataratas puede interferir con la transmisión de la señal óptica, un examinador experimentado es capaz de obtener imágenes nítidas a pesar de la presencia de una esclerosis cristalina relativamente densa $(19,20)$. Publicaciones previas han comunicado que aunque las MER no siempre pueden ser identificadas con certeza en las imágenes de OCT, casi constantemente aparece una distorsión de la arquitectura foveolar normal (21-25). La detección de dicha distorsión debería conducir al examinador a un estudio más detallado de la imagen topográfica, en la que casi siempre se puede identificar al menos parcialmente la MER (fig. 2).

En este estudio, hemos encontrado una prevalencia de MER del 15,6\% en pacientes referidos para cirugía de cataratas; una prevalencia elevada, similar a la descrita en pacientes afáquicos y pseudofáquicos en los estudios epidemiológicos mencionados anteriormente $(2,3,12-14)$. El hecho de que la mayoría de estas MER no eran evidentes en la oftalmoscopía, junto con el hecho de que no se detectaron nuevas MER en los 6 meses de seguimiento tras la cirugía de cataratas, nos conducen a plantear que 
la frecuencia de MER no detectadas puede ser mayor de lo que se pensaba. Nuestro estudio se diseñó con un período de seguimiento de 6 meses porque en el trabajo de Jahn y colaboradores, la incidencia de nuevas MER no aumentó de forma significativa entre los 6 y los 12 meses de seguimiento (15).

La OCT parece ser un método de detección de MER más preciso que la fotografía de fondo de ojo, especialmente en pacientes con esclerosis cristalina. Nuestros resultados sugieren la posibilidad de que muchas MER previamente asociadas a la facoemulsificación no son producidas por la cirugía, sino simplemente mejor diagnosticadas una vez que se obtiene una imagen nítida del fondo de ojo. La mayoría de estas membranas serían del tipo RCM, que en su mayoría son asintomáticas y probablemente pasan desapercibidas después de la cirugía de catarata.

Somos conscientes del limitado número de pacientes incluido en nuestro estudio y de la dificultad de extraer conclusiones de él. Sin embargo, sugiere que la cirugía de catarata, o al menos las técnicas actuales de facoemulsificación mínimamente agresivas, no están directamente relacionadas con el desarrollo de MER en los primeros 6 meses tras la cirugía. Serían necesarios estudios más amplios para determinar de manera definitiva si la facoemulsificación es un desencadenante para el desarrollo de MER.

\section{BIBLIOGRAFÍA}

1. Appiah AP, Hirose T. Secondary causes of premacular fibrosis. Ophthalmology 1989; 96: 389-392.

2. Mitchell P, Smith W, Chey T, Wang JJ, Chang A. Prevalence and associations of epiretinal membranes. The Blue Mountains Eye Study, Australia. Ophthalmology 1997; 104: 1033-1040.

3. Klein R, Klein BE, Wang Q, Moss SE. The epidemiology of epiretinal membranes. Trans Am Ophthalmol Soc 1994; 92: 403-425.

4. Wiznia RA. Natural history of idiopathic preretinal macular fibrosis. Ann Ophthalmol 1982; 14: 876-878.

5. Wise GN. Relationship of idiopathic preretinal macular fibrosis to posterior vitreous detachment. Am J Ophthalmol 1975; 79: 358-362.

6. Hirokawa H, Jalkh AE, Takahashi M, Takahashi M, Trempe CL, Schepens CL. Role of the vitreous in idiopathic preretinal macular fibrosis. Am J Ophthalmol 1986; 101: 166-169.

7. Appiah AP, Hirose T, Kado M. A review of 324 cases of idiopathic premacular gliosis. Am J Ophtalmol 1988; 106: 533-535.
8. Wiznia RA. Posterior vitreous detachment and idiopatic preretinal macular gliosis. Am J Ophthalmol 1986; 102: 196-198.

9. Wise GN. Clinical features of idiopathic preretinal macular fibrosis. Schoenberg Lecture. Am J Ophthalmol 1975; 79: 349-357.

10. Pearlstone AD. The incidence of idiopathic preretinal macular gliosis. Ann Ophthalmol 1985; 17: 378-380.

11. McCarty DJ, Mukesh BN, Chikani V, Wang JJ, Mitchell P, Taylor H, et al. Prevalence and associations of epiretinal membranes in the visual impairment project. Am J Ophthalmol 2005; 140: 288-294.

12. Fraser-Bell S, Guzowski M, Rochtchina E, Wang JJ, Mitchell P. Five-year cumulative incidence and progression of epiretinal membranes: The Blue Mountains Eye Study. Ophthalmology 2003; 110: 34-40.

13. Fraser-Bell S, Ying-Lai M, Klein R, Varma R; Los Angeles Latino Eye Study. Prevalence and association of epiretinal membranes in latinos: the Los Angeles Latino Eye Study. Invest Ophthalmol Vis Sci 2004; 45: 1732-1736.

14. Miyazaki $M$, Nakamura $H$, Kubo M, Kiyohara $Y$, Iida $M$, Ishibashi $M$, et al. Prevalence and risk factors for epiretinal membranes in a Japanese population: the Hisayama study. Graefes Arch Clin Exp Ophthalmol 2003; 241: 642646.

15. Jahn CE, Minich V, Moldaschel S, Stahl B, Jedelhauser P, Kremer $G$, et al. Epiretinal membranes after extracapsular cataract surgery (1). J Cataract Refract Surg 2001; 27: 753-760.

16. Chylack LT Jr, Wolfe JK, Singer DM, Leske MC, Bullimore MA, Bailey IL, et al. The Lens Opacities Classification System III. The Longitudinal Study of Cataract Study Group. Arch Ophthalmol 1993; 111: 831-836.

17. Huang D, Swanson EA, Lin CP, Schuman JS, Stinson WG, Chang $W$, et al. Optical coherence tomography. Science 1991; 254: 1178-1181.

18. Hee MR, Izatt JA, Swanson EA, Huang D, Schuman JS, Lin CP, et al. Optical coherence tomography of the human retina. Arch Ophthalmol 1995; 113: 325-332.

19. Hee MR. Artifacts in optical coherence tomography topography maps. Am J Ophthalmol 2005; 139: 154-155.

20. Ray R, Stinnett SS, Jaffe GJ. Evaluation of image artifact produced by optical coherence tomography of retinal pathology. Am J Ophthalmol 2005; 139: 18-29.

21. Wilkins JR, Puliafito CA, Hee MR, Duker JS, Reichel E, Coker JG, et al. Characterization of epiretinal membranes using optical coherence tomography. Ophthalmology 1996; 103: 2142-2151.

22. Azzolini C, Patelli F, Codenotti M, Pierro L, Brancato R. Optical coherence tomography of idiopathic epiretinal macular membrane surgery. Eur J Ophthalmol 1999; 9: 206-211.

23. Massin P, Allough C, Haouchine B, Metge F, Paques M, Tangui L, et al. Optical coherence tomography of idiopathic macular epiretinal membranes before and after surgery. Am J Ophthalmol 2000; 130: 732-739.

24. Mori K, Gehlbach P, Sano A, Deguchi T, Yoneya S. Comparison of epiretinal membranes of differing pathogenesis using optical coherence tomography. Retina 2004; 24: 5762.

25. Hassenstein A, Scholz, F, Richard G. OCT bei epiretinal Gliosis. Ophthalmologe 2005; 102: 127-132. 